\title{
X-RAY INDUCED CHANGES IN BIOCHEMICAL AND HISTOCHEMICAL PARAMETERS IN THE TESTIS OF MALE WILD INDIAN HOUSE RAT, RATTUS RATTUS
}

\author{
Kalyan Brata Santra ${ }^{1}$ and Chanchal Kumar Manna ${ }^{2}$ \\ ${ }^{1}$ Department of Zoology, Daulatpur High School, Daulatpur, Dakshin Dinajpur, West Bengal, India \\ ${ }^{2}$ Endocrinology Laboratory, Department of Zoology, University of Kalyani, Kalyani-741235, \\ West Bengal, India \\ Accepted 20 December 2009
}

\begin{abstract}
To observe the complementary effects, whole body of the male wild Indian house rat, Rattus rattus was irradiated with X-rays at $100 \mathrm{r}, 200 \mathrm{r}, 300 \mathrm{r}$ and $500 \mathrm{r}$ (single dose) doses. The changes in the histophysiological parameters of the testis were observed after 2, 7, 15 and 30 days of post treatment. Determination of biochemical parameters: ascorbic acid, cholesterol, acid and alkaline phosphatases in the testicular tissues indicated that concentrations of ascorbic acid and cholesterol ( 2 to 15 days post treatment) had increased while acid and alkaline phosphatases (2-30 days) had decreased. Changes in these parameters were observed in all four X- ray treated groups $(100 \mathrm{r}, 200 \mathrm{r}, 300 \mathrm{r}$ and $500 \mathrm{r})$. The histochemical study of the different X-ray treated groups clearly showed the intense accumulation of lipid substances and depletion of acid, alkaline phosphatases, $\Delta^{5}-3 \beta-\mathrm{HSDH}$ and $17 \beta-\mathrm{HSDH}$ in the testicular tissues of 7 to 30 day - post treated groups exposed to $300 \mathrm{r}$ and $500 \mathrm{r} \mathrm{X}$-irradiation. In view of the changes observed in the testicular tissues, it is likely that a single dose of $\mathrm{X}$ - ray may cause certain histophysiological changes in the testis of wild rodents at least for certain periods. As a single dose of Xrays of 200r or 300r was sufficient to cause sterility in male rats, this may be included as a controlling agent that adversely effect the fertility of the rodent pests. Possible mode of action is discussed.
\end{abstract}

Key Words: rodent pest, acid and alkaline phosphatases, lipid, fertility, spermatogenesis

\section{INTRODUCTION}

Alberts-Schonberg's observation in 1903 showed that radiation causes a rapid regression of the mammalian testis. Numerous studies have shown that the testis is one of the most important radiosensitive organs in the body (Ellis and Berliner, 1969). The testis decreases in size and a gradual cessation of spermatogenesis occur due to radiation (Santra and Manna, 2002; Heller, 1948). Ellinger (1957) reported the arrest of cell division, followed by the disappearance of various tubular elements in the following order : spermatogonia, spermatocytes, spermatid and spermatozoa.

Craig et al. (1961) reported that, of the 3 different doses of X-ray (200 r, $300 \mathrm{r}$ and $500 \mathrm{r}$ ) to which mice and rats were exposed, the dose $300 \mathrm{r}$ produced a period of complete sterility in both mice and rats. Hopkinson et al. (1978) reported the progressive reduction of total tubular area due to shrinkage of cytoplasm, spermatocyte and spermatid nuclear area due to $\mathrm{X}$-irradiation at $300 \mathrm{r}$.

Abbott (1959) concluded that the endocrine functions of the testis remain unchanged after radiation. Fogg and Cowing (1951) cited evidence proposed by other workers for an altered pituitary-testis axis. Some workers have shown that endogenic substances or pituitary gonadotrophins have a radioprotective action on the testis (Johnson and Witschi, 1963; Binhammer, 1967). Gunn et al. (1960; 1961), have since shown that androgen secretion by the testis is altered after radiation. The steroid biotransformations can be altered by irradiation and this was first shown by Rosenfeld et al. (1955) and Berliner et al. (1962), for the adrenal glands. Testicular steroid biotransformations were shown to be altered by various modes of Xirradiation by Shikita and Tamaoki (1965) and Simpson and Ellis (1967).

The adverse effect of X-rays on the testicular tissues has been well studied in many

*Corresponding author's email: kalyan_117@rediffmail.com 
mammalian species. But there is no substantial information on the effects of X-ray on the testicular histochemistry and biochemistry of the wild rats to our knowledge. In order to fill this gap in information, the effect of X-rays on the testicular tissues of the wild Indian house rat (Rattus rattus) was studied.

\section{MATERIALS AND METHODS}

In the present study 120 adult male wild Indian house rats (Rattus rattus) were used. Prior to the experiment, rats were collected from the surrounding fields of the Kalyani University and were maintained under normal laboratory conditions (L 12h: D 12h). To determine the effect of radiation on the testis, rats were exposed to four different doses of X-ray $100 \mathrm{r}$, 200 r, $300 \mathrm{r}$ and $500 \mathrm{r}$. After exposure of X-ray, animals were maintained under laboratory conditions. Observations were made in four successive phases. Six rats from each group (control, 100 r, 200 r, 300 r, 500 r) were autopsied after 2 days $\left(1^{\text {st }}\right.$ phase $), 7$ days $\left(2^{\text {nd }}\right.$ phase), 15 days ( $3^{\text {rd }}$ phase) and 30 days $\left(4^{\text {th }}\right.$ phase).

For the quantitative estimation of various biochemical components eg. total cholesterol (Zarrow et al., 1964), ascorbic acid (Nino and Prasad, 1980), acid and alkaline phosphatases (Walter and Schutt, 1974) within the testis of control and the four experimental groups, at least six treated rats were used. At autopsy, the testis was removed, weighed in a torsion balance, homogenised in different required media for the extraction of cholesterol, ascorbic acid, acid and alkaline phosphatase. After homogenisation they were centrifuged and the supernatants were used to estimate the biochemical components. The measurements were made at relevant wavelengths using a spectrophotometer (Spectronic 20 Genesys). After assessing for each biochemical component, the data were analysed using Student's ' $t$ ' test.

The reference to the procedure used for cytochemical analysis is given against each of the constituents. For lipids (Kay and Whitehead, 1941), $\Delta^{5}$-3 $\beta$-hydroxysteroid dehydrogenase (Wattenberg, 1958 modified by Bilaspuri and Guraya, 1984), 17 $\beta$-hydroxysteroid dehydrogenase (Pearson and Grose, 1959 modified by Bilaspuri and Guraya, 1984), alkaline phosphatase Butcher and Chayan, 1966 and acid phosphatase (Bitensky, 1963). At the end of cytochemical procedures, tissue sections were mounted in glycerine jelly and observations were made under light microscopy. HIstochemical changes within the testis of control and experimental rats were carefully examined and their changes were recorded. Photomicrographs were taken from stained sections.

\section{RESULTS}

\section{Biochemical components}

Testicular extracts of control rats contained very low concentrations of ascorbic acid and cholesterol (Tables 1 and 2). The concentration of acid and alkaline phosphatases were very high (Tables 1 and 2). The different biochemical components in the testicular extract of 2 day post-irradiated rats are given in Table 1. The concentration of cholesterol had increased with an increasing in dosage from $100 \mathrm{r}$ to $500 \mathrm{r}$. The concentration of ascorbic acid in the $100 \mathrm{r}$ and $200 \mathrm{r}$ treated rats has also increased compared to the control. The concentration of acid phosphatase in the $300 \mathrm{r}$ and $500 \mathrm{r}$ treated and the concentration of alkaline phosphatase in the $100 \mathrm{r}, 200 \mathrm{r}, 300 \mathrm{r}$ and $500 \mathrm{r}$ treated rats are quite low in the 2 day- post treated rats compared to the control (Table 1). In the 7 day- post treated rats the concentration of ascorbic acid in the 200 $\mathrm{r}, 300 \mathrm{r}$ and $500 \mathrm{r}$ X-ray irradiated groups and cholesterol in the $200 \mathrm{r}, 300 \mathrm{r}, 500 \mathrm{r}$ groups had increased than the control rats. The concentration of ascorbic acid higher than in the 2 day-treated rats (Table 1). The concentration of acid phosphatase in the $300 \mathrm{r}$ and $500 \mathrm{r}$ groups and alkaline phosphatase in all four treated groups had decreased. The concentration of cholesterol in the $200 \mathrm{r}, 300 \mathrm{r}$ and $500 \mathrm{r}$ treated groups in 15 day- post treated rats was very high compared to the control. The maximum concentration was in the $300 \mathrm{r}$ group. In the $100 \mathrm{r}, 300 \mathrm{r}$ and $500 \mathrm{r}$ groups the concentration of ascorbic acid in the testicular extract was higher (Table 2) but the concentration of alkaline phosphatase in the 100 r, $200 \mathrm{r}, 300 \mathrm{r}$ and $500 \mathrm{r}$ groups and alkaline phosphatase in the $100 \mathrm{r}, 200 \mathrm{r}, 300 \mathrm{r}$ and $500 \mathrm{r}$ groups had decreased in comparison to the control. The concentration of alkaline phosphatase in the $500 \mathrm{r}$ group was very low than in the control group. In 30 day- post treated rats, the concentration of ascorbic acid was closer to the control group. But the concentration of cholesterol in the $300 \mathrm{r}$ and $500 \mathrm{r}$ groups was higher than in the control group. The activity of 
alkaline phosphatase in the $100 \mathrm{r}, 200 \mathrm{r}, 300 \mathrm{r}$ and $500 \mathrm{r}$ groups and acid phosphatase in the $500 \mathrm{r}$ group had decreased in the 30 day - post treated rats in comparison to the control (Table $2)$.

Table 1. Effect of different dosages of radiation (X-ray) on biochemical components of testes 2 and 7 days after exposure of adult male Rattus rattus.

\begin{tabular}{|c|c|c|c|c|}
\hline \multirow{2}{*}{$\begin{array}{l}\text { Control and } \\
\text { treatment Groups } \\
\text { (No. exposed) }\end{array}$} & \multicolumn{2}{|c|}{ (mmol/100mg fresh testicular tissue) } & \multicolumn{2}{|c|}{ (mg/100mg fresh testicular tissue) } \\
\hline & $\begin{array}{c}\text { Acid } \\
\text { phosphatase }\end{array}$ & $\begin{array}{c}\text { Alkaline } \\
\text { phosphatase }\end{array}$ & Ascorbic acid & Cholesterol \\
\hline Control $(6) * * *$ & $0.694 \pm 0.054 *$ & $0.312 \pm 0.013$ & $0.018 \pm 0.0009$ & $0.529 \pm 0.02$ \\
\hline 100r- 2 days (6) & $0.770 \pm 0.086$ & $0.192 \pm 0.014$ & $0.019 \pm 0.0004$ & $0.720 \pm 0.09$ \\
\hline $200 r-2$ days (6) & $0.669 \pm 0.014$ & $0.189 \pm 0.024$ & $0.022 \pm 0.001$ & $0.774 \pm 0.02$ \\
\hline $300 r-2$ days (6) & $0.547 \pm 0.033$ & $0.169 \pm 0.007$ & $0.017 \pm 0.0009$ & $0.793 \pm 0.07$ \\
\hline 500r- 2 days (6) & $0.452 \pm 0.036$ & $0.236 \pm 0.04$ & $0.018 \pm 0.0008$ & $0.808 \pm 0.03$ \\
\hline \multirow[t]{2}{*}{$\begin{array}{l}\text { F-Table } 5 \% \\
\text { F- Calculated } \\
\text { CD- value at } 5 \%\end{array}$} & $\begin{array}{l}2.76 \\
6.14 \\
0.12\end{array}$ & $\begin{array}{l}2.76 \\
6.27 \\
0.06\end{array}$ & $\begin{array}{c}2.76 \\
4.84 \\
0.002\end{array}$ & $\begin{array}{l}2.76 \\
4.32 \\
0.17\end{array}$ \\
\hline & $\mathrm{T}_{1}>\mathrm{C}>\mathrm{T}_{2}>\mathrm{T}_{3}>\mathrm{T}_{5} * *$ & $\mathrm{C}>\mathrm{T}_{5}>\mathrm{T}_{1}>\mathrm{T}_{2}>\mathrm{T}_{3}$ & $\mathrm{~T}_{2}>\underline{\mathrm{T}_{1}}>\mathrm{C}=\mathrm{T}_{5}>\mathrm{T}_{3}$ & $\mathrm{~T}_{5}>\mathrm{T}_{3}>\mathrm{T}_{2}>\mathrm{T}_{1}>\mathrm{C}$ \\
\hline Control (6) & $0.568 \pm 0.016$ & $0.246 \pm 0.013$ & $0.019 \pm 0.001$ & $0.670 \pm 0.05$ \\
\hline 100r- 7 days (6) & $0.591 \pm 0.054$ & $0.167 \pm 0.01$ & $0.018 \pm 0.001$ & $0.499 \pm 0.03$ \\
\hline 200r- 7 days (6) & $0.595 \pm 0.047$ & $0.165 \pm 0.026$ & $0.025 \pm 0.002$ & $0.749 \pm 0.04$ \\
\hline $300 r-7$ days $(6)$ & $0.490 \pm 0.026$ & $0.147 \pm 0.013$ & $0.028 \pm 0.002$ & $0.783 \pm 0.04$ \\
\hline 500r- 7 days (6) & $0.417 \pm 0.013$ & $0.123 \pm 0.015$ & $0.038 \pm 0.009$ & $0.783 \pm 0.03$ \\
\hline \multirow{4}{*}{$\begin{array}{l}\text { F-Table } 5 \% \\
\text { F- Calculated } \\
\text { CD- value at } 5 \%\end{array}$} & 2.76 & 2.76 & 2.76 & 2.76 \\
\hline & 4.73 & 7.85 & 3.60 & 10.29 \\
\hline & 0.10 & 0.02 & 0.01 & 0.11 \\
\hline & $\underline{\mathrm{T}_{2}>\mathrm{T}_{1}>\mathrm{C}>} \underline{\mathrm{T}_{3}>\mathrm{T}_{5}}$ & $\mathrm{C}>\underline{\mathrm{T}_{1}>\mathrm{T}_{2}}>\mathrm{T}_{3}>\mathrm{T}_{5}$ & $\mathrm{~T}_{5}>\underline{\mathrm{T}_{3}>\mathrm{T}_{2}>\mathrm{C}}>\mathrm{T}_{1}$ & $\underline{\mathrm{T}_{5}}=\mathrm{T}_{3}>\mathrm{T}_{2}>\mathrm{C}>\mathrm{T}_{1}$ \\
\hline
\end{tabular}


Table 2. Effect of different dosages of radiation (X-ray) on biochemical components of testes 15 and 30 days after exposure of adult male Rattus rattus.

\begin{tabular}{|c|c|c|c|c|}
\hline \multirow{2}{*}{$\begin{array}{l}\text { Control and } \\
\text { treatment Groups } \\
\text { (No. exposed) }\end{array}$} & \multicolumn{2}{|c|}{ (mmol/100mg fresh testicular tissue) } & \multicolumn{2}{|c|}{ (mg/100mg fresh testicular tissue) } \\
\hline & Acid phosphatase & $\begin{array}{c}\text { Alkaline } \\
\text { phosphatase }\end{array}$ & Ascorbic acid & Cholesterol \\
\hline Control (6)*** & $0.630 \pm 0.036^{*}$ & $0.242 \pm 0.002$ & $0.019 \pm 0.009$ & $0.627 \pm 0.05$ \\
\hline $100 r-15$ days $(6)$ & $0.550 \pm 0.077$ & $0.122 \pm 0.019$ & $0.020 \pm 0.001$ & $0.575 \pm 0.03$ \\
\hline $200 r-15$ days (6) & $0.493 \pm 0.029$ & $0.124 \pm 0.005$ & $0.019 \pm 0.001$ & $0.698 \pm 0.04$ \\
\hline $300 r-15$ days $(6)$ & $0.504 \pm 0.027$ & $0.119 \pm 0.006$ & $0.021 \pm 0.0004$ & $1.010 \pm 0.41$ \\
\hline 500r- 15 days $(6)$ & $0.461 \pm 0.02$ & $0.111 \pm 0.002$ & $0.023 \pm 0.002$ & $0.701 \pm 0.02$ \\
\hline \multirow[t]{2}{*}{$\begin{array}{l}\text { F-Table } 5 \% \\
\text { F- Calculated } \\
\text { CD- value at } 5 \%\end{array}$} & $\begin{array}{l}2.76 \\
2.31 \\
0.12\end{array}$ & $\begin{array}{c}2.76 \\
36.67 \\
0.03\end{array}$ & $\begin{array}{c}2.76 \\
1.65 \\
0.004\end{array}$ & $\begin{array}{c}2.76 \\
18.13 \\
0.11\end{array}$ \\
\hline & $\underline{\mathrm{C}>\mathrm{T}_{1}}>\mathrm{T}_{3}>\mathrm{T}_{2}>\mathrm{T}_{5} * *$ & $C>\underline{T_{2}>T_{1}>T_{3}>T_{5}}$ & $\mathrm{~T}_{5}>\mathrm{T}_{3}>\mathrm{T}_{1}>\mathrm{T}_{2}=\mathrm{C}$ & $\mathrm{T}_{3}>\mathrm{T}_{5}>\mathrm{T}_{2}>\mathrm{C}>\mathrm{T}_{1}$ \\
\hline Control (6) & $0.599 \pm 0.029$ & $0.274 \pm 0.019$ & $0.018 \pm 0.001$ & $0.537 \pm 0.04$ \\
\hline $100 r-30$ days $(6)$ & $0.643 \pm 0.052$ & $0.177 \pm 0.005$ & $0.017 \pm 0.0007$ & $0.453 \pm 0.03$ \\
\hline $200 r-30$ days (6) & $0.608 \pm 0.034$ & $0.152 \pm 0.026$ & $0.018 \pm 0.001$ & $0.595 \pm 0.05$ \\
\hline $300 r-30$ days $(6)$ & $0.538 \pm 0.021$ & $0.143 \pm 0.006$ & $0.021 \pm 0.002$ & $0.846 \pm 0.06$ \\
\hline 500r- 30 days (6) & $0.366 \pm 0.044$ & $0.130 \pm 0.016$ & $0.021 \pm 0.0008$ & $0.910 \pm 0.15$ \\
\hline \multirow{4}{*}{$\begin{array}{l}\text { F-Table } 5 \% \\
\text { F- Calculated } \\
\text { CD- value at } 5 \%\end{array}$} & 2.76 & 2.76 & 2.76 & 2.76 \\
\hline & 8.60 & 12.39 & 1.90 & 6.41 \\
\hline & 0.11 & 0.05 & 0.004 & 0.24 \\
\hline & $\underline{\mathrm{T}_{1}>\mathrm{T}_{2}>\mathrm{C}>\mathrm{T}_{3}>\mathrm{T}_{5}}$ & $\mathrm{C}>\underline{\mathrm{T}_{1}>\mathrm{T}_{2}>\mathrm{T}_{3}>\mathrm{T}_{5}}$ & $\underline{\mathrm{T}_{5}}=\mathrm{T}_{3}>\mathrm{T}_{2}=\mathrm{C}>\mathrm{T}_{1}$ & $\underline{\mathrm{T}_{5}>\mathrm{T}_{3}}>\underline{\mathrm{T}_{2}}>\mathrm{C}>\mathrm{T}_{1}$ \\
\hline
\end{tabular}

** Underline indicates insignificant difference among the groups at $5 \%$ level

$\mathrm{C}, \mathrm{T}_{1}, \mathrm{~T}_{2}, \mathrm{~T}_{3}$, and $\mathrm{T}_{5}$ are the mean value of control, 100r, 200r, 300r and 500r of X-ray irradiated groups respectively.

\section{Histochemical components}

The control group contained lesser amount of sudanophilic lipids (Fig. 1C). The intensity of $\Delta^{5}$-3 $\beta$-hydroxysteroid dehydrogenase, $17 \beta$ hydroxysteroid dehydrogenase, alkaline phosphatase (Fig. 1A) and acid (Fig. 1E) phosphatase were quite high (Table $3 a, b, c, d$ ). The histochemical distribution of different components in the testicular tissue of 2 day post treated rats are presented in Table 3 a. Sudanophilic lipid granules were found with higher intensities especially in the $300 \mathrm{r}$ and 500 $r$ irradiated rats than in control rats. The intensity of acid and alkaline phosphatases and $17 \beta-$ HSDH of $300 \mathrm{r}$ and $500 \mathrm{r}$ groups had also decreased, but no remarkable change in $\Delta^{5}-3 \beta$ HSDH within the seminiferous tubules were found in the 2 day- post treated rats compared to the control. In comparison to the control rats, a significant depletion in the activity of acid and alkaline phosphatases, $\Delta^{5}-3 \beta-H S D H$ and $17 \beta-$ HSDH were found in the 7 days post treated 
groups (Table 3b). Sudanophilic lipid granules are found with higher intensities in the $300 \mathrm{r}$ (Fig. 1D) and $500 \mathrm{r}$ post irradiated groups of 7 day - post treated rats than in the control. Reduction in the intensity of $\Delta^{5}-3 \beta$-HSDH, $17 \beta$ HSDH, acid (Fig. 1F) and alkaline (Fig. 1B) phosphatases in the $300 \mathrm{r}$ and $500 \mathrm{r}$ groups were found following 15 days of post irradiation in comparison to the control, but the sudanophilic lipid granules regained towards normal levels (Table 3c). In 30 day post treated rats a decrease in the intensity of acid and alkaline phosphatases were observed in the $300 \mathrm{r}$ and $500 \mathrm{r}$ treated groups. But the activity of sudanophilic lipids, $\Delta^{5}-3 \beta-\mathrm{HSDH}$ and $17 \beta \mathrm{HSDH}$ were closer towards the control level (Table $3 \mathrm{~d}$ ).

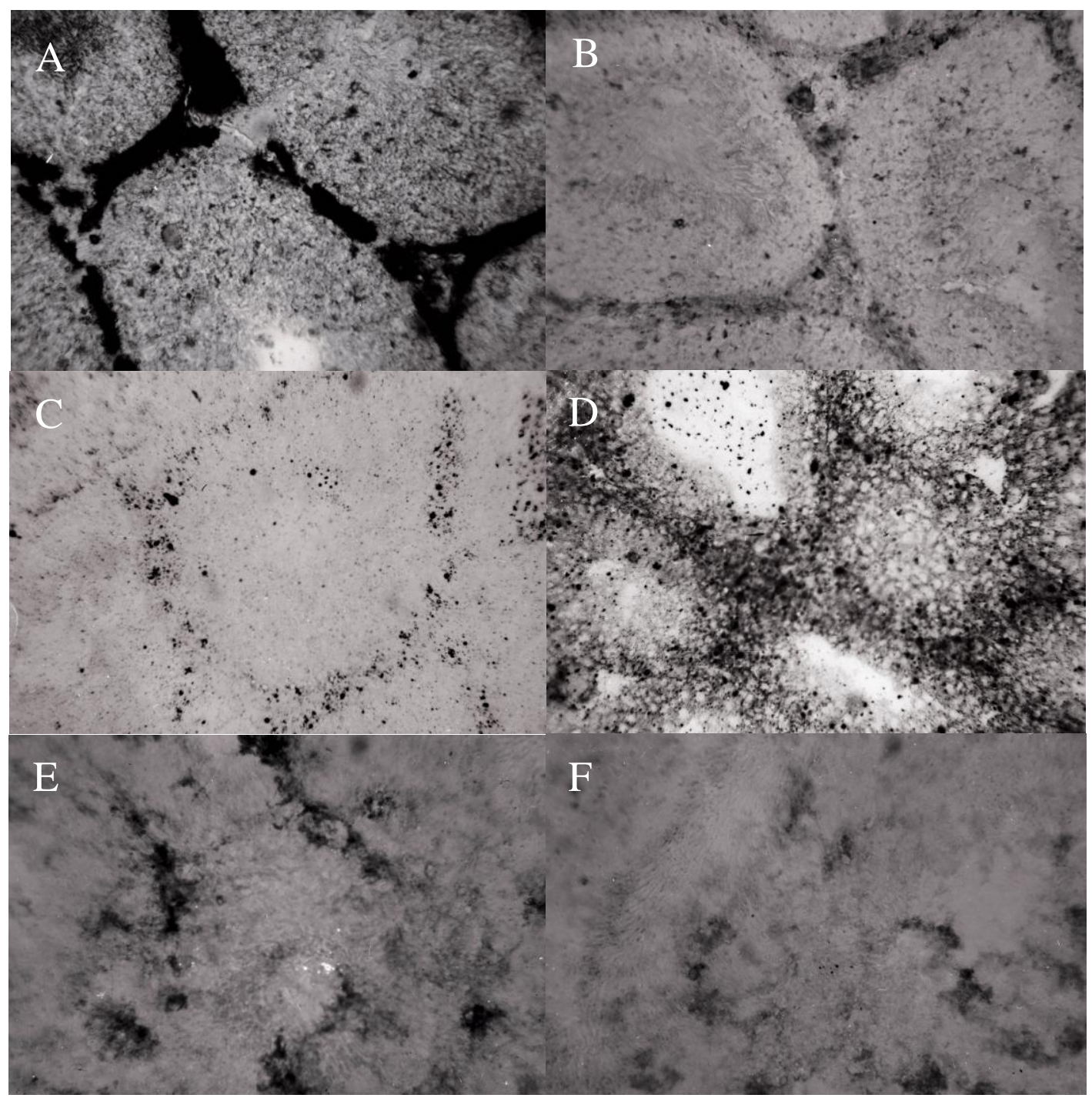

Figure 1. (A) Cryocut section of testis in control rats showing strong alkaline phosphatase activity within interstitial cells and basement membrane $(\times 200)$. (B) Very low alkaline phosphatase activity within interstitial cells and basement membrane of the seminiferous tubules in $300 \mathrm{r} X$-irradiated rats after 15 days $(\times 200)$. (C) Section of testis of control rats showing the distribution of Sudanophilic lipids in interstitial cells, basement membrane and spermatogonial cells. $(\times 200)$. (D) Section of the testis of 300r $\mathrm{X}$-irradiated rats 7 days after treatment showing increased lipid granules within seminiferous tubular region. Interstitial cells show similar lipid content as in control rats. ( $\times 200)$. (E) Strong acidphosphatase activity within interstitial cells of testis in control rats $(\times 200)$. (F) Low acid phosphatase activity within interstitial cells of testicular sections in rats 15 days after 300r $\mathrm{X}$-irradiation $(\times 200)$. 
Table 3 a. Histochemical changes in the testis of male Rattus rattus 2 days after treatment with different doses of $\mathrm{X}$-rays.

\begin{tabular}{|c|c|c|c|c|c|c|}
\hline Tested for & Regions & Control & $\begin{array}{l}2 \text { days } \\
-100 r\end{array}$ & $\begin{array}{l}2 \text { days } \\
-200 r\end{array}$ & $\begin{array}{l}2 \text { days } \\
-300 r\end{array}$ & $\begin{array}{l}2 \text { days } \\
-500 r\end{array}$ \\
\hline \multirow{3}{*}{$\begin{array}{l}\text { Sudan } \\
\text { III \& IV }\end{array}$} & i) Basement membrane & ++ & ++ & ++ & ++ & +++ \\
\hline & ii) Seminiferous tubule & + & + & + & ++ & ++ \\
\hline & iii) Interstitium & ++ & ++ & ++ & ++ & ++ \\
\hline \multirow{3}{*}{$\begin{array}{l}\text { Acid } \\
\text { phosphatase }\end{array}$} & i) Basement membrane & ++ & ++ & ++ & ++ & ++ \\
\hline & ii) Seminiferous tubule & \pm & \pm & \pm & \pm & \pm \\
\hline & iii) Interstitium & ++ & ++ & ++ & ++ & ++ \\
\hline \multirow{3}{*}{$\begin{array}{l}\text { Alkaline } \\
\text { phosphatase }\end{array}$} & i) Basement membrane & + & + & \pm & \pm & + \\
\hline & ii) Seminiferous tubule & + & + & \pm & \pm & + \\
\hline & iii) Interstitium & + & + & + & + & + \\
\hline \multirow{3}{*}{$\begin{array}{l}\text { 17 } \beta \text {-hydroxy } \\
\text { steroid } \\
\text { dehydrogenase }\end{array}$} & i) Basement membrane & ++ & ++ & + & + & + \\
\hline & ii) Seminiferous tubule & ++ & ++ & + & + & + \\
\hline & iii) Interstitium & ++ & ++ & ++ & ++ & ++ \\
\hline \multirow{3}{*}{$\begin{array}{l}\Delta^{5}-3 \beta \text { - hydroxy } \\
\text { steroid } \\
\text { dehydrogenase }\end{array}$} & i) Basement membrane & +++ & ++ & ++ & + & + \\
\hline & ii) Seminiferous tubule & + & + & + & \pm & \pm \\
\hline & iii) Interstitium & +++ & ++ & ++ & + & \pm \\
\hline
\end{tabular}

Intensity of reaction: + : Positive ; - : Negative ; ++ : Moderate ; +++ : Highly positive or intensed; \pm : Not significant

Table 3 b. Histochemical changes in the testis of male Rattus rattus 7 days after treatment with different doses of $\mathrm{X}$-rays.

\begin{tabular}{|c|c|c|c|c|c|c|}
\hline Methods & Regions & Control & $\begin{array}{l}7 \text { days } \\
-100 \mathrm{r}\end{array}$ & $\begin{array}{l}7 \text { days } \\
-200 r\end{array}$ & $\begin{array}{l}7 \text { days } \\
-300 r\end{array}$ & $\begin{array}{l}7 \text { days } \\
-500 \mathrm{r}\end{array}$ \\
\hline $\begin{array}{l}\text { Sudan } \\
\text { III \& IV }\end{array}$ & $\begin{array}{l}\text { i) Basement membrane } \\
\text { ii) Seminiferous tubule } \\
\text { iii) Interstitium }\end{array}$ & $\begin{array}{l}++ \\
+ \\
++\end{array}$ & $\begin{array}{l}++ \\
+ \\
++\end{array}$ & $\begin{array}{l}++ \\
++ \\
++\end{array}$ & $\begin{array}{l}++ \\
+++ \\
++\end{array}$ & $\begin{array}{l}+++ \\
++ \\
+++\end{array}$ \\
\hline $\begin{array}{l}\text { AAcid } \\
\mathrm{p} \\
\text { phosphatases }\end{array}$ & $\begin{array}{l}\text { i) Basement membrane } \\
\text { ii) Seminiferous tubule } \\
\text { iii) Interstitium }\end{array}$ & $\begin{array}{l}++ \\
\pm \\
++\end{array}$ & $\begin{array}{l}++ \\
\pm \\
+\end{array}$ & $\begin{array}{l}++ \\
\pm \\
\pm\end{array}$ & $\begin{array}{l}+ \\
- \\
-\end{array}$ & $\begin{array}{l}+ \\
- \\
-\end{array}$ \\
\hline $\begin{array}{l}\text { Alkaline } \\
\text { phosphatase }\end{array}$ & $\begin{array}{l}\text { i) Basement membrane } \\
\text { ii) Seminiferous tubule } \\
\text { iii) Interstitium }\end{array}$ & $\begin{array}{l}+ \\
+ \\
+\end{array}$ & $\begin{array}{l}+ \\
+ \\
\pm\end{array}$ & $\begin{array}{l} \pm \\
- \\
\pm\end{array}$ & $\begin{array}{l} \pm \\
- \\
\pm\end{array}$ & $\begin{array}{l} \pm \\
- \\
-\end{array}$ \\
\hline $\begin{array}{l}17 \beta \text {-hydroxy } \\
\text { steroid } \\
\text { dehydrogenase }\end{array}$ & $\begin{array}{l}\text { i) Basement membrane } \\
\text { ii) Seminiferous tubule } \\
\text { iii) Interstitium }\end{array}$ & $\begin{array}{l}++ \\
++ \\
++\end{array}$ & $\begin{array}{l}++ \\
+ \\
++\end{array}$ & $\begin{array}{l}+ \\
+ \\
+\end{array}$ & $\begin{array}{l} \pm \\
+ \\
+\end{array}$ & $\begin{array}{l} \pm \\
+ \\
\pm\end{array}$ \\
\hline $\begin{array}{l}\Delta^{5}-3 \beta- \\
\text { hydroxy } \\
\text { steroid } \\
\text { dehydrogenase }\end{array}$ & $\begin{array}{l}\text { i) Basement membrane } \\
\text { ii) Seminiferous tubule } \\
\text { iii) Interstitium }\end{array}$ & $\begin{array}{l}+++ \\
+ \\
+++\end{array}$ & $\begin{array}{l}++ \\
+ \\
++\end{array}$ & $\begin{array}{l}++ \\
+ \\
++\end{array}$ & $\begin{array}{l}++ \\
+ \\
++\end{array}$ & $\begin{array}{l}++ \\
+ \\
+\end{array}$ \\
\hline
\end{tabular}


Table 3 c. Histochemical changes in the testis of male Rattus rattus 15 days after treatment with different doses of X-rays

\begin{tabular}{|c|c|c|c|c|c|c|}
\hline Methods & Regions & Control & $\begin{array}{l}15 \text { days } \\
-100 \mathrm{r}\end{array}$ & $\begin{array}{l}15 \text { days } \\
-200 r\end{array}$ & $\begin{array}{l}15 \text { days } \\
-300 \mathrm{r}\end{array}$ & $\begin{array}{l}15 \text { days } \\
-500 \mathrm{r}\end{array}$ \\
\hline $\begin{array}{l}\text { Sudan } \\
\text { III \& IV }\end{array}$ & $\begin{array}{l}\text { i) Basement membrane } \\
\text { ii) Seminiferous tubule } \\
\text { iii) Interstitium }\end{array}$ & $\begin{array}{l}++ \\
+ \\
++\end{array}$ & $\begin{array}{l}++ \\
+ \\
++\end{array}$ & $\begin{array}{l}++ \\
+ \\
++\end{array}$ & $\begin{array}{l}+++ \\
+ \\
+++\end{array}$ & $\begin{array}{l}+++ \\
++ \\
+++\end{array}$ \\
\hline $\begin{array}{l}\text { Acid } \\
\text { phosphatase }\end{array}$ & $\begin{array}{l}\text { i) Basement membrane } \\
\text { ii) Seminiferous tubule } \\
\text { iii) Interstitium }\end{array}$ & $\begin{array}{l}++ \\
\pm \\
++\end{array}$ & $\begin{array}{l}++ \\
\pm \\
++\end{array}$ & $\begin{array}{l}++ \\
+ \\
+\end{array}$ & $\begin{array}{l} \pm \\
- \\
\pm\end{array}$ & $\begin{array}{l} \pm \\
- \\
-\end{array}$ \\
\hline $\begin{array}{l}\text { Alkaline } \\
\text { phosphatase }\end{array}$ & $\begin{array}{l}\text { i) Basement membrane } \\
\text { ii) Seminiferous tubule } \\
\text { iii) Interstitium }\end{array}$ & $\begin{array}{l}+ \\
+ \\
+\end{array}$ & $\begin{array}{l}+ \\
+ \\
+\end{array}$ & $\begin{array}{l}+ \\
+ \\
+\end{array}$ & $\begin{array}{l}+ \\
\pm \\
-\end{array}$ & $\begin{array}{l}+ \\
\pm \\
-\end{array}$ \\
\hline $\begin{array}{l}17 \beta \text {-hydroxy } \\
\text { steroid } \\
\text { dehydrogenase }\end{array}$ & $\begin{array}{l}\text { i) Basement membrane } \\
\text { ii) Seminiferous tubule } \\
\text { iii) Interstitium }\end{array}$ & $\begin{array}{l}++ \\
++ \\
++\end{array}$ & $\begin{array}{l}++ \\
+ \\
++\end{array}$ & $\begin{array}{l}+ \\
+ \\
+\end{array}$ & $\begin{array}{l} \pm \\
\pm \\
+\end{array}$ & $\begin{array}{l} \pm \\
\pm \\
+\end{array}$ \\
\hline $\begin{array}{l}\Delta^{5}-3 \beta \text { - hydroxy } \\
\text { steroid } \\
\text { dehydrogenase }\end{array}$ & $\begin{array}{l}\text { i) Basement membrane } \\
\text { ii) Seminiferous tubule } \\
\text { iii) Interstitium }\end{array}$ & $\begin{array}{l}+++ \\
+ \\
+++\end{array}$ & $\begin{array}{l}++ \\
+ \\
++\end{array}$ & $\begin{array}{l}++ \\
+ \\
+\end{array}$ & $\begin{array}{l}+ \\
+ \\
+\end{array}$ & $\begin{array}{l}+ \\
+ \\
\pm\end{array}$ \\
\hline
\end{tabular}

Intensity of reaction: + : Positive ;,$:$ Negative $;++$ : Moderate $;+++:$ Highly positive or intensed; \pm : Not significant

Table $3 \mathrm{~d}$. Histochemical changes in the testis of male Rattus rattus 30 days after treatment with different doses of $\mathrm{X}$-rays.

\begin{tabular}{|c|c|c|c|c|c|c|}
\hline Methods & Regions & Control & $\begin{array}{l}30 \text { days } \\
-100 r\end{array}$ & $\begin{array}{l}30 \text { days } \\
-200 r\end{array}$ & $\begin{array}{l}30 \text { days } \\
-300 r\end{array}$ & $\begin{array}{l}30 \text { days } \\
-500 r\end{array}$ \\
\hline \multirow{3}{*}{$\begin{array}{l}\text { Sudan } \\
\text { III \& IV }\end{array}$} & i) Basement membrane & ++ & ++ & ++ & ++ & ++ \\
\hline & ii) Seminiferous tubule & + & + & + & + & ++ \\
\hline & iii) Interstitium & ++ & ++ & ++ & ++ & + \\
\hline \multirow{3}{*}{$\begin{array}{l}\text { Acid } \\
\text { phosphatase }\end{array}$} & i) Basement membrane & ++ & ++ & ++ & + & \pm \\
\hline & ii) Seminiferous tubule & \pm & \pm & + & + & \pm \\
\hline & iii) Interstitium & ++ & ++ & + & + & + \\
\hline \multirow{3}{*}{$\begin{array}{l}\text { Alkaline } \\
\text { phosphatase }\end{array}$} & i) Basement membrane & + & + & + & + & + \\
\hline & ii) Seminiferous tubule & + & + & + & + & \pm \\
\hline & iii) Interstitium & + & + & + & + & + \\
\hline \multirow{3}{*}{$\begin{array}{l}\text { 17 } \beta \text {-hydroxy } \\
\text { steroid } \\
\text { dehydrogenase }\end{array}$} & i) Basement membrane & ++ & ++ & ++ & + & + \\
\hline & ii) Seminiferous tubule & ++ & ++ & ++ & + & + \\
\hline & iii) Interstitium & ++ & ++ & ++ & ++ & + \\
\hline \multirow{3}{*}{$\begin{array}{l}\Delta^{5}-3 \beta \text { - hydroxy } \\
\text { steroid } \\
\text { dehydrogenase }\end{array}$} & i) Basement membrane & +++ & +++ & +++ & ++ & + \\
\hline & ii) Seminiferous tubule & + & + & + & + & \pm \\
\hline & iii) Interstitium & +++ & +++ & ++ & ++ & \pm \\
\hline
\end{tabular}

Intensity of reaction: + : Positive ; - : Negative ; ++ : Moderate $;+++:$ Highly positive or intensed; \pm : Not significant 


\section{DISCUSSION}

The present investigation demonstrates structural damages to the testis, of the adult wild male Indian house rat (Rattus rattus) after X-ray irradiation at doses of 100 r, 200 r, $300 \mathrm{r}$ and 500 $\mathrm{r}$. From the histochemical study, high amount of lipid materials were observed in X-ray treated groups. In the $300 \mathrm{r}$ and $500 \mathrm{r}$ groups 7 and 15 days of post treatment, the accumulation of lipid granules were maximum. Lipid accumulation in mammals within the testicular tissues, especially in the Sertoli cells due to local irradiation or after high dose of ionizing radiation has been described by Lacy et al. (1965); Collins and Lacy (1969). High amount of lipid granules in the Sertoli cells of mammals seem to be due to the phagocytosed lipid materials of the degenerating germ cells (Lacy, 1960). It is also known that the lipid inclusion in Sertoli cell is under the control of FSH secretion from the pituitary (Kerr and de Kretser, 1975). Serum FSH has been shown to be related to the germinal cell component, particularly spermatogonial numbers (de Kretser et al., 1974). Irradiation and local heat application to the testis are known to disrupt spermatogenesis and cause an increase in the lipid inclusion in Sertoli cells of rat (Collins and Lacy, 1969) and to be associated with elevated levels of FSH, presumably resulting from a decrease in the feedback signal from the testis (Kerr and de Kretser, 1975).

Some alteration in the steroid dehydrogenase enzymes $\left(\Delta^{5}-3 \beta-\mathrm{HSDH}\right.$ and $\left.17 \beta-\mathrm{HSDH}\right)$ has been observed in rats exposed to various doses of X-rays. But when the lipids and steroid enzymes are taken together, some inverse relationship has been observed in the testicular tissues due to X-ray treatment. The lower activity levels of the dehydrogenase enzymes within the interstitial cells of various X-ray treated rats seem to be due to the spermatogenetically inactive gonads. Although there are some controversy, most are of the opinion that in mammals, the Leydig cells are not affected by radiation, i.e., they are radioresistant (Ellis, 1970; Setchell, 1978). Both lipids and steroid dehydrogenases are usually known to show an inverse relationship. Higher content of lipids and lower activity levels of dehydrogenases are characteristic of steroidogenically inactive gonads.

Not only steroid enzymes but the activities of phosphatase enzymes were also found to decrease in various X-ray treated groups of rats (both biochemically and histochemically). The alkaline phosphatase is said to be a histochemical marker for primordial germ cells of various species, including rat (Mc Alpine, 1955) and mouse (Mintz, 1957). It is known that alkaline phosphatase enzyme is required for the synthesis of glycogen, which in turn apparently participates in the metabolic process of spermatogenesis (Sohval, 1958). Mann (1964) reported an intense activity of acid phosphatase in the seminal plasma of several mammalian species including humans. Seminal and prostatic acid phosphatase have been associated with the nutrition of spermatozoa (Mann, 1964; Serrano et al., 1976) with their fertilizing ability (Singer et al., 1980). Szego (1972, 1974) reported that lysosomal acid phosphatase of the rat preputial gland is implicated with the mediation of steroids and hypophysial gonadotrophins.

It is also known in the present investigation that ascorbic acid in the testicular tissues increased due to X-ray irradiation. In mammals, ascorbic acid has been found to exert an inhibitory role on steroidogenesis (Kitabchi, 1967). Also ascorbic acid is a known catalyst for both lipid peroxidation and alteration of unsaturated fatty acid composition (Shimizu, 1970). Hence, the involvement of ascorbic acid in the process of steroidogenesis in the testis of control and treated groups may be taken into consideration. It is well established that cholesterol plays an important role in the inhibition or stimulation of sperm formation in the testis and is the primary substrate for androgen biosynthesis. Increased testicular cholesterol content after the X-ray irradiation is also suggested for reduction in steroid production (Mohanty and Chainy, 1988). Elevated testicular cholesterol level may be implicated with the altered lipid metabolism, which is frequently associated with testicular atrophy (Santra and Manna, 2002).

It has been suggested (Ellis and Berliner, $1969)$ that $17 \beta$-ol-dehydrogenase (oxidase) is located in both tubules and interstitial elements and diminishes progressively during the interval after irradiation. This phenomenon is directly related to the destructive changes associated with the depopulation of the seminiferous tubules. They also suggested the destruction of the germinal epithelium with direct or whole body irradiation, that affect both the reduction of androstenedione to testosterone and the oxidation of testosterone to androstenedione. Thus, a very dynamic relationship seems to exist 
between androgen synthesis and spermatogenesis. Closely associated with the changes in androgen synthesis is the effect of radiation on DNA synthesis. Thus, it can be said that although Sertoli and Leydig cells are histologically insensitive they are " biochemically radiosensitive" (Ellis and Berliner, 1969).

The findings infer that X-ray irradiation causes significant damages to the testis. Considering the changes observed it can be stated that the single dose of X-ray i.e. 300r is sufficient for the suppression of spermatogenetic activity at least for few days in the wild Indian house rat, Rattus rattus. It also suggests that exposure to a single dose of X-ray of 200r or 300r may affect the fertility of the rodent pests and thereby bring about their control.

\section{ACKNOWLEDGEMENTS}

The authors are indebted to UGC, of the Government of India for financial support (No.F.3-19/98, SR-II). We are grateful to the Central Research Institute for Jute and Allied Fibres, Barrackpore, W.B., for providing X-ray facilities.

\section{REFERENCES}

Abbott, C. R. (1959). The effect of X-irradiation on the secretory capacity of the testis. $J$. Endocrinol., 19: 33-43.

Alberts-Schonberg, H. E. (1903). Uber eine bisher unbekannte Wirkung der Rontgenstrahlen auf den Organismus der Tier. Muench. Med. Wschr., 50: 1859-1860.

Berliner, D., Berliner, M. L. and Dougherty, T. F. (1962). The effect of chronic irradiation by internally deposited radionuclides on corticosteroid biosynthesis. In: 'Some aspects of internal irradiation'. Dougherty, T. F. et al. (eds). pp.179-186. Pergamon Press, Oxford.

Binhammer, R. T. (1967). Effect of increased endogenous gonadotrophin on testis of irradiated immature and mature rats. Radiat. Res., 30: 676686.

Bitensky, L. (1963). Modifications of the Gomori acid phosphatase technique for controlled temperature frozen sections. Quart. J. Micros. Sci., 104: 193-196.

Butcher, R. G. and Chayan, J. (1966). Quantitative studies on the alkaline phosphatase reaction . J. Roy. Micros. Soc., 85: 111-117.

Chang, C. H., Angellis, D. and Fishman, W. H. (1980). Presence of the rare D-variant, heat stable, placental type alkaline phosphatase in normal human testes . Cancer Res., 40: 15061510 .

Collins, P. and Lacy, D. (1969). Studies on the structure and function of the mammalian testis. II. Cytological and histochemical observations on the testis of the rat after a single exposure to heat applied for different lengths of time. Proc. Roy. Soc. B., 172: 17-38.

Craig, A. W., Fox, B. W. and Jackson, H. (1961). Effect of radiation on male mouse and rat fertility. J. Repord. Fert., 2: 466-472.

de Kretser, D. M., Burger, H. G. and Hudson, B. (1974). The relationship between germinal cells and serum FSH levels in males with infertility. $J$. Clin. Endocrinol. Metab., 38 : 793-797.

Ellinger, F. (1957) Medical Radiation Biology. Charles, C. Thomas (Eds), Springfield, Illinois. Pp. 274-301.

Ellis, L. C. (1970). "Radiation effects" . In : 'The Testis'. Johnson, A. D., Gomes, W. R. and Van Demark, N. L. (eds). Vol. III. pp. 333-376. Academic Press, New York and London.

Ellis, L. C. and Berliner, D. L. (1969). Alterations in testicular androgen biosynthesis as related changes in spermatogenesis induced by ionizing radiations. In : 'The Gonads'. Mc Kerns, K.W. ( eds ). pp. 739-783. Appleton, New York.

Fogg, L. C. and Cowing, R. F. (1951). The changes in cell morphology and histochemistry of the testes following irradiation and their relation to other induced testicular changes. I. Quantitative random sampling of germinal cells at intervals following direct irradiation. Cancer Res., 11: 23-28.

Gunn, S. A., Gould, T. C. and Anderson, W. A. D. (1960). The effect of X-irradiation on the morphology and function of the rat testis. Am. J. Path., 37: 203-213. 
Gunn, S. A., Gould, T. C. and Anderson, W. A. D. (1961). The effect of microwave radiation on morphology and function of rat testes . Lab. Invest., 10: 301-314.

Heller, M. (1948). The testis, in histopathology of irradiation from external and internal sources, W. Bloom, ed. McGraw Hill Book Company, Inc., New York, National Nuclear Energy series .Div. IV, Vol. 221.Chap.12.

Hopkinson, C. N. R., Dulisch, B., Gauss, G., Hilscher, W. and Hirschhauser, C. (1978). The effect of local irradiation on testicular histology and plasma hormone levels in the male rat. Acta Endocrinol., (Copenh) 87: 413-423.

Johnson, D. C. and Witschi, E. (1963). Response of X-irradiated and cryptorchid testes to exogenous gonadotrophins in parabiotic rats. $J$. Endocrinol., 27: 193-199.

Kay, W. W. and Whitehead, R. (1941). Sudan III and IV methods for neutral fats. In: 'Histochemistry-Theoretical and Applied'. $2^{\text {nd }}$ edition . Pearse, A. G. E. (ed). pp 853-854.

Kerr, J. B. and de Kretser, D. M. (1975). Cyclic variations in Sertoli cell lipid content throughout the spermatogenic cycle in the rat. J. Reprod. Fert., 43: 1-8.

Kitabchi, A. E. (1967). Ascorbic acid in steroidogenesis. Nature, (Lond). 215: 13851386.

Lacy, D. (1960). Light and electron microscopy and its use in the study of factors influencing spermatogenesis in the rat. J. Roy. Micros. Soc., 79: 209-225.

Lacy, D., Lofts, B., Kinson, G., Hopkins, D. and Dott, H. (1965). Sertoli cells and steroid synthesis . Gen. Comp. Endocrinol., 5: 693 (abstr).

Mann, T. (1964). "The biochemistry of semen and of the male reproductive tract". Methuen and Co. Ltd., London.

Mc Alpine, R. J. (1955). Alkaline glycerophosphatase in the developing adrenals, gonads and reproductive tract of the white rat. Anat. Rec., 121: 407-408.

Mintz, B. (1957). Embryological development of primordial germ cells in the mouse: influence of a new mutation . W. J. Embryol. Exp. Morphol., 5: 396-403.

Mohanty, N. and Chainy, G. B. N. (1988) Seasonal and hormones-induced changes in testicular functions of the musk shrew (Suncus murinus L.). Indian J. exp. Biol., 26: 274-276.

Nino, H. V. and Prasad, A. S. (1980). Ascorbic acid (Vitamin C) . In : "Vitamins and trace elements". Gradwohl's Clinical Laboratory Methods and Diagnosis. Vol.1. Sonnenwirth, A. C. and Jarett, L. ( eds ). pp. 370-372. The C. V. Mosby Comp., St. Louis, Toronto, London.

Pearson, B. and Grose, F. (1959). Histochemical demonstration of 17 $\beta$-hydroxysteroid dehydrogenase by use of a tetrazolium salt. Proc. Soc. Exptl. Biol. Med., 100: 636-638.

Rosenfeld, G., Ungar, F., Dorfman, R. I. and Pincus, G. (1955). Irradiation and adrenal steroidogenesis. Steroid transformations by irradiated isolated perfused calf adrenals. Endocrinology, 56: 24-29.

Santra, K. B. and Manna, C. K. (2002). X-ray induced changes of some haematological and histological parameters of the testis in the male wild Indian house rat, Rattus rattus. Proceedings of the national seminar on recent advances in molecular Physiology, Univ. of Kalyani, held on 4-6 February, 174-180

Serrano, J. A., Shannon, W. A. Jr., Sternberger, N. J., Wasserkrug, H. L., Serrano, A. A. and Seligman, A. M. (1976). The cytochemical demonstration of prostate acid phosphatase using a new substrate, phosphorylcholine . $J$. Histochem. Cytochem., 24: 1046-1056.

Setchell, B. P. (1978). Naturally occurring and induced disfunctions of the testis. In: 'The mammalian testis'. Setchell, B. P. ( ed ). pp. 359. Paul Eleck, London.

Shimizu, K. (1970). Effects of ascorbic acid on the side chain cleavage of cholesterol. Biochem. Biophys. Acta., 210 : 333-340.

Simpson, C. G. and Ellis, L. C. (1967). The direct effects of $\mathrm{X}$-irradiation of the biosynthesis of androgens by rat testicular tissue in vitro. Radiat. Res., 31: 139-148.

Singer, R., Barnet, M., Allalcuf, U., Schwartzman, S., Sagiv, M., Landau, B., Segenreich, E. and Servadio, C. (1980). Some 
properties of acid and alkaline phosphatase in seminal fluid and isolated sperm. Arch. Androl., 5: 195-199.

Sohval, A. R. (1958). The anatomy and endocrine physiology of the male reproductive system. In : 'The Endocrinology of reproduction'. Velardo, J. T. (Ed). pp. 243-312. Oxford University Press, New York.

Szego, C. M. (1972). Lysosomal membrane stabilization and antiestrogen action in specific hormonal target cells. Gynae. Invest., 3: 63 .

Szego, C. M. (1974). The lysosomes as a mediator of hormone action. Recent Prog. Horm. Res., 30: 171-233.
Walter, K. and Schutt, C. (1974). Acid and alkaline phosphatase in serum (Two point method). In : 'Methods of Enzymatic Analysis'. Vol. 2. Bergmeyer, H. U, ( ed ). pp. 856-860. Academic Press, New York, Sanfrancisco, London.

Wattenberg, L. W. (1958). Microscopic histochemical demonstration of steroid $3 \beta$-oldehydrogenase in tissue sections. J. Histochem. Cytochem. , 6: 225-232.

Zarrow, M. X., Yochim, J. M. and Mc Carthy, J. T. (1964). Experimental endocrinology: A source book of basic techniques. Academic Press, New York. 